VOL. 69 (2004) [69-86]

\title{
ON REGULARITY PRESERVATION IN A SEMIGROUP
}

\author{
J.B.HICKEY
}

\begin{abstract}
We consider certain subsets of a semigroup $S$, defined mainly by conditions involving regularity preservation. In particular, the regular base $\mathrm{B}(S)$ of $S$ may be regarded as a generalisation of the zero ideal in a semigroup with zero; if it is non-empty then $S$ is $E$-inversive. The other subsets considered are related in a natural way either to $\mathrm{B}(S)$ or to the set $\mathrm{RP}(S)$ of regularity-preserving elements in $S$. In a regular semigroup (equipped with the Hartwig-Nambooripad order) each of these subsets contains either minimal elements only or maximal elements only. The relationships between the subsets are discussed, and some characterisations of completely simple semigroups are obtained.
\end{abstract}

\section{INTRODUCTION}

For a semigroup $S$ with a regular element, the set $\operatorname{RP}(S)$ of regularity-preserving elements of $S$ was introduced in [8] and further studied in $[2,12]$. If the subset $\operatorname{RRP}(S)$ of regular elements in $\mathrm{RP}(S)$ is non-empty, then it is a completely simple subsemigroup of $S$ and can be regarded as a generalisation to $S$ of the group of units in a monoid [8]. In a regular semigroup $S$, all the elements of $\operatorname{RP}(S)(=\operatorname{RRP}(S))$ are maximal in $S$ under the Hartwig-Nambooripad partial order $[7,16]$ and all belong to a maximum $\mathcal{J}$-class. Thus $\operatorname{RP}(S)$, if non-empty, can be said to be at the 'upper end' of a regular semigroup $S$.

Section 2 below is devoted to notation and preliminaries. In sections 3 and 4 we consider certain subsets of a semigroup $S$ that are similar to $\mathrm{RP}(S)$ or are related to it in a natural way. These are defined mainly by conditions involving regularity preservation. In particular, for a semigroup $S$ with a regular element, we define a subset $\mathrm{B}(S)$ of $S$ that can be regarded as an analogue of $\operatorname{RP}(S)$ lying at the 'lower end' of $S$ : if it is non-empty, it is a completely simple subsemigroup of $S$ and forms a minimum ideal for $S$ (Theorem 3.7); also, it reduces to $\{0\}$ in the case when $S$ has a zero element. Further, a semigroup $S$ with the property that $\mathrm{B}(S)$ is non-empty must be $E$-inversive (Corollary 4.7). The remaining subsets under discussion are closely related to (and may coincide with) either $\mathrm{RP}(S)$ or $\mathrm{B}(S)$, and we determine the relationships that exist, in general,

Received 30th June, 2003

Copyright Clearance Centre, Inc. Serial-fee code: 0004-9727/04 $\$ A 2.00+0.00$. 
between them (Lemma 3.6, Lemma 3.8, Corollary 3.12, Theorems 4.2, 4.4 and 4.8). In a regular semigroup each of these subsets contains either maximal elements only or minimal elements only.

Section 5 is concerned with regular semigroups. Here, elements that are weakly cancellable on $S$ are seen to be maximal in $S$ (Corollary 5.4). The set of such elements is considered in relation to the other sets of maximal elements under discussion (Theorem 5.3 ), and some characterisations of completely simple semigroups are obtained (Theorems 5.5 and 5.6). At the 'lower end', we have that $\mathrm{B}(S)$ coincides with the set of minimal elements in $S$ (Theorem 5.1).

Section 6 is devoted to examples.

\section{Preliminaries}

We use the notation and terminology of $[6,10,11]$ throughout. In particular, $\operatorname{Reg}(S)$ will denote the set of regular elements in a semigroup $S$.

We begin by recalling some ideas and results from $[17, \mathbf{1}, \mathbf{8 , 2}, \mathbf{7}, \mathbf{1 6}]$. Let $a$ be an element of a (multiplicative) semigroup $S$. By a pre-inverse [post-inverse] of a we mean an element $b \in S$ such that $a b a=a[b a b=b][8]$. We denote the set of pre-inverses [post-inverses] of $a$ by $\operatorname{Pre}(a)[\operatorname{Post}(a)]$. Thus we have that $V(a)=\operatorname{Pre}(a) \cap \operatorname{Post}(a)$, where, as usual, $V(a)$ denotes the set of inverses of $a$ in $S$. (It should be remarked here that post-inverses are often called weak inverses in the literature.) Clearly we have that $\operatorname{Post}(a) \subseteq \operatorname{Reg}(S)$ for all $a \in S$.

By a mididentity [or middle unit or midunit] in a semigroup $S$ we mean an element $u \in S$ such that $x u y=x y$ for all $x, y \in S[17,1,8]$.

If $S$ is a semigroup and $a \in S$, the binary operation o defined on the set $S$ by $x \circ y=x a y$ is associative; the resulting semigroup is denoted by $(S, a)$ and is called a variant of $S$. Variants have been studied in $[8,2,9,12]$ and form a special case of the semigroups studied in [4].

For a regular semigroup $S$, the Hartwig-Nambooripad partial order $\leqslant$ on $S[7,16]$ may be formulated in several ways (see, for example, [10, Section 1.4]). One such is the following: for $x, y \in S$,

$$
x \leqslant y \Leftrightarrow(\exists e, f \in E(S)) \quad x=e y=y f
$$

We note that restricting this to $E(S) \times E(S)$ gives the natural partial order on $E(S)$, so that if $e$ and $f$ are idempotents in $S$, then

$$
e \leqslant f \Leftrightarrow e=e f=f e
$$

Further, if $S$ is an inverse semigroup then $\leqslant$ reduces to the natural order relation on $S$. 
The Hartwig-Nambooripad order may also be formulated in terms of variants [8, Theorem 5.1]: for $x, y \in S$,

$$
x \leqslant y \Leftrightarrow(\exists a \in S) \quad x, y \in E((S, a)) \text { and } x=x \circ y=y \circ x \text { in }(S, a) .
$$

LEMMA 2.1. [2, Lemma 2.1] Let $x, y$ be elements of a regular semigroup $S$ and let $y^{\prime}$ be a pre-inverse of $y$. Then

$$
x \leqslant y \Leftrightarrow x \in E\left(\left(S, y^{\prime}\right)\right) \text { with } x=x \circ y=y \circ x \text { in }\left(S, y^{\prime}\right) .
$$

Throughout the paper, when we say that an element $a$ of a regular semigroup $S$ is minimal [maximal] in $S$, we shall mean that $a$ is minimal [maximal] in $S$ under the above partial order.

Returning to arbitrary semigroups $S$, we see that every element that is regular in a variant $(S, a)$ must also be regular in $S$, but that the converse is not true. It is natural then to make the following definition [8]: if $a, x$ are elements of a semigroup $S$, then we say that a preserves the regularity of $x$ (or simply that a preserves $x$ ) if $x$ is regular in $(S, a)$. We may note that if, for $a, x \in S$, we have that $a$ preserves $x$, then $x$ must already be regular in $S$.

Suppose that, for a semigroup $S$, the set $\operatorname{Reg}(S)$ is not empty. If $a \in S$ preserves every element of $\operatorname{Reg}(S)$ then we say that $a$ is a regularity-preserving element in $S$. The set of such elements in $S$ is denoted by $\operatorname{RP}(S)$. The set of regular regularity-preserving elements in $S$ is denoted by $\operatorname{RRP}(S)$. We can regard $\mathrm{RP}(S)$ (when $S$ has a mididentity) and $\operatorname{RRP}(S)$ as generalisations to $S$ of the group of units in a monoid [8, Section 4]. In particular, in an arbitrary semigroup $S$, the subset $\operatorname{RRP}(S)$, if non-empty, forms a completely simple subsemigroup of $S$ that reduces to the group of units of $S$ when $S$ is a monoid [8, Theorem 4.4 and 4.7]. In a regular semigroup $S$, with $\operatorname{RP}(S) \neq \emptyset$, the elements of $\operatorname{RP}(S)[=\mathrm{RRP}(S)]$ are maximal in $S$ (under the Hartwig-Nambooripad order) [2, Corollary 2.4]; also, as shown by Khan and Lawson [12, p. 360], all such elements belong to a maximum $\mathcal{J}$-class. We may thus regard $\operatorname{RP}(S)$, if non-empty, as lying at the 'upper end' of a regular semigroup $S$.

If $S$ is completely simple then each variant of $S$ is regular [8, Lemma 3.4], so each element of $S$ preserves all the elements of $S$. It follows that $\operatorname{RP}(S)=S$ here.

The following simple observation will be useful to us throughout the paper.

LEMMA 2.2. Let $S$ be a semigroup. If $a, b \in S$ are such that $b a b=b$, then $a$ preserves $b$.

PROOF: If $b a b=b$, then $b$ is an idempotent in $(S, a)$ and hence is regular there. $\quad$

It follows that an element of a semigroup preserves each of its post-inverses and is preserved by each of its pre-inverses. 
3. REgUlaRITY PRESERVATION AND SOME RELATED SUBSETS OF A SEMIGROUP

We begin with an easy generalisation of [12, Section 2, Proposition 3].

Lemma 3.1. Let $S$ be a semigroup and let $x, y$ be elements of $S$. Then $x$ preserves $y \Leftrightarrow y$ is regular and $y x \mathcal{R} y \mathcal{L} x y$.

Proof: Suppose $x$ preserves $y$. Then $y=y \circ z \circ y$ in $(S, x)$ for some $z \in S$, that is, $y=y x z x y$. It follows that $y$ is regular and that $y x \mathcal{R} y \mathcal{L} x y$.

Conversely, suppose $y$ is regular and that $y x \mathcal{R} y \mathcal{L} x y$. Then, for some $z, w \in S^{1}$, we have $y x z=y=w x y$. Let $y^{\prime} \in \operatorname{Pre}(y)$. Then

$$
y=y y^{\prime} y=(y x z) y^{\prime}(w x y)=y \circ\left(z y^{\prime} w\right) \circ y \text { in }(S, x) .
$$

Thus $x$ preserves $y$.

The next result is similar to [8, Lemma 4.3].

LEMmA 3.2. Let $S$ be a semigroup.

(i) If $a \in S$ is such that a preserves itself, then $H_{a}$ is a subgroup of $S$.

(ii) Let $b$ and $c$ be elements of $S$ such that $b \in c S \cap S c$. If $b$ preserves an element $x \in S$ then $c$ must also preserve $x$.

(iii) Let $b$ and $c$ be regular elements of $S$ such that $c \in b S \cap S b$. If an element $x \in S$ preserves $b$ then $x$ must also preserve $c$.

PROOF: The proofs of (i) and (ii) are easy generalisations of the proof of [8, Lemma 4.3].

We prove (iii). Let $b$ and $c$ be as given and let $x \in S$ preserve $b$. Then $b=b x w x b$ for some $w \in S$. Also $c=b s=t b$ for some $s, t \in S$ and so $c=b x w x c=c x w x b$. But $c=c z c$ for some $z \in S$, since $c$ is regular, and so $c=(c x w x b) z(b x w x c)$, giving that $x$ preserves $c$, as required.

Now let $S$ be a semigroup and let $a, b \in S$. We shall call $(a, b)$ a mutually preserving pair if $a$ and $b$ preserve each other. Clearly, if $(a, b)$ is a mutually preserving pair then so is $(b, a)$.

LEMMA 3.3. Let $S$ be a semigroup and let $(a, b)$ be a mutually preserving pair in $S$. Then

(i) $b a \mathcal{R} b \mathcal{L} a b$ and $a b \mathcal{R} a \mathcal{L} b a$,

(ii) $a b a=a \Leftrightarrow b a b=b$.

Proof: (i) follows from Lemma 3.1. To prove (ii), suppose that $a b a=a$. Then, by (i) and Green's Lemma [11, Lemma 2.2.1] applied in $S, \rho_{b}\left|L_{a}, \rho_{a}\right| L_{b}$ are mutually inverse bijections from $L_{a}$ onto $L_{b}$ and from $L_{b}$ onto $L_{a}$, respectively. Thus $b a b=b$. We have now proved that $a b a=a \Rightarrow b a b=b$, and the result follows from interchanging $a$ and $b$. 
Let $S$ be a semigroup and let $a \in S$. We shall say that $a$ is a universal pre-inverse [universal post-inverse] (element) for $S$ if $a$ is a pre-inverse [post-inverse] of every element of $S$, that is if $b a b=b[a b a=a]$ for all $b \in S$.

Let $\operatorname{UPre}(S)$, UPost $(S)$ denote the set of universal pre-inverses, universal postinverses, respectively, in $S$. Thus, for $a \in S$,

$$
a \in \mathrm{UPre}(S) \Leftrightarrow \operatorname{Post}(a)=S, \quad a \in \operatorname{UPost}(S) \Leftrightarrow \operatorname{Pre}(a)=S .
$$

Clearly, a universal post-inverse for $S$ must be a regular element of $S$, and a semigroup with a universal pre-inverse is necessarily regular. Further, $\operatorname{UPre}(S) \subseteq \operatorname{RP}(S)$, since a universal pre-inverse must preserve each of its post-inverses (by Lemma 2.2), that is, must preserve every element of $S$.

It is straightforward to show that if $u$ is either a universal pre-inverse or a universal post-inverse for $S$ and if $u$ belongs to a subgroup $H$ of $S$, then $H$ must be trivial. We use this observation in the proof of the following result.

LEMMA 3.4. Let $u$ be either a universal pre-inverse or a universal post-inverse for a semigroup $S$. Then $u$ is idempotent and the $\mathcal{H}$-class $H_{u}$ is trivial.

Proof: In either case $u$ is a pre-inverse of itself, so $u$ preserves itself. By Lemma $3.2, H_{u}$ is a subgroup of $S$ and so must be trivial. It follows that $u^{2}=u$, proving the result.

One would expect the presence of a universal pre-inverse to have a restricting effect on a semigroup, and the next two results confirm this.

Lemma 3.5. Let $S$ be a semigroup with a universal pre-inverse element. Then, for all $x, y \in S$, we have

$$
\text { (i) } x^{3}=x^{2}, \quad \text { (ii) }(x y)^{2} x=(x y) x, \quad \text { (iii) } x^{2} \in E(S) \text {. }
$$

Proof: Let $u$ be a universal pre-inverse in $S$ and let $x, y \in S$. The proofs of (i) and (ii) follow from considering the products $x^{2} u x^{2}$ and $(x y x) u(x y x)$, respectively, and (iii) follows from (i).

LEMMA 3.6. Let $S$ be a semigroup with a universal pre-inverse element. Then $\operatorname{UPre}(S)=\operatorname{RP}(S)$ and $\operatorname{RP}(S)$ is a rectangular band. If, in addition, $S$ has a mididentity, then every element of $\operatorname{RP}(S)$ is a mididentity for $S$, and $S$ is a band.

Proof: Let $u$ be a universal pre-inverse for $S$ ( so $S$ is a regular semigroup). As remarked earlier, we have $\mathrm{UPre}(S) \subseteq \operatorname{RP}(S)$. Conversely, let $a \in \operatorname{RP}(S)$. To show that $a \in \operatorname{UPre}(S)$ we must show that $x a x=x$ for all $x \in S$. So let $x \in S$. We have $a=a u a$ and $x=$ xayax for some $y \in S$. But axaxa $=$ axa by Lemma 3.5 (ii). Thus

$$
x a y(a x a x a) y a x=x a y(a x a) y a x,
$$


that is, $x a x=x$. Thus $a \in \operatorname{UPre}(S)$, so $\operatorname{RP}(S) \subseteq \operatorname{UPre}(S)$, and hence UPre $(S)=\operatorname{RP}(S)$. Also, $\operatorname{RP}(S)$ is a completely simple subsemigroup of $S$ by [8, Theorem 4.4]. It is a band by Lemma 3.4, and so is a rectangular band.

Suppose further that $S$ has a mididentity element $a$. If $b \in \operatorname{RP}(S)[=\operatorname{UPre}(S)]$ then $b$ is a pre-inverse of $a$, so $b$ is a mididentity for $S$ by [8, Corollary 3.2]. Thus every element of $\operatorname{RP}(S)$ is a mididentity. Finally, let $u \in \operatorname{UPre}(S)$, as above, and let $x \in S$. Then $x u x=x$. But $u$ is a mididentity for $S$, so we have $x^{2}=x$. It follows that $S$ is a band.

We shall later (in Lemma 3.8) have results on UPost $(S)$ similar to those on UPre $(S)$ contained in Lemma 3.6. For now we note that if a semigroup $S$ has a zero element 0 , then 0 is a universal post-inverse for $S$ and $\operatorname{UPost}(S)=\{0\}$. More generally, if $S$ has a right [left] zero element $z$, then $z \in \operatorname{UPost}(S)$ and $\operatorname{UPost}(S)$ coincides with the set of right [left] zero elements of $S$, as may readily be proved.

We now consider an object that may be regarded as a 'lower end' analogue of RP $(S)$.

Let $S$ be a semigroup with $\operatorname{Reg}(S) \neq \emptyset$. We define the regular base of $S$, denoted by $\mathrm{B}(S)$, to be the set of elements of $S$ that are preserved by every element of $S$. Thus, for $b \in S$,

$$
b \in \mathrm{B}(S) \Leftrightarrow b \in \operatorname{Reg}(S, x) \text { for eyery } x \in S .
$$

We note that $\mathrm{B}(S) \subseteq \operatorname{Reg}(S)$. If $S$ has a zero element 0 , then $0 \in \mathrm{B}(S)$, and any element $b \in \mathrm{B}(S)$ must be preserved by 0 , so $b=0$. Thus $\mathrm{B}(S)=\{0\}$ in this case.

We have remarked in Section 2 that if $S$ is completely simple then every variant of $S$ is regular. So, in this case, each element of $S$ is preserved by all the elements of $S$. It follows that $\mathrm{B}(S)=S$ here.

THEOREM 3.7. Let $S$ be a semigroup with $\mathrm{B}(S) \neq \emptyset$. Then

(i) $\mathrm{B}(S)$ is a minimum ideal for $S$.

(ii) $\mathrm{B}(S)$ is a completely simple subsemigroup of $S$.

Proof: (i) Let $b \in \mathrm{B}(S)$ and let $s \in S$. We first show that $s b \in \mathrm{B}(S)$. Let $x \in S$. Then $b$ is preserved by $x s$, and so $b=b(x s) z(x s) b$ for some $z \in S$. Thus $s b=s b . x(s z) x . s b$. So $s b$ is preserved by $x$. It follows that $s b \in \mathrm{B}(S)$. Similarly, using the fact that $b$ is preserved by $s x$, we find that $b s \in \mathrm{B}(S)$. Thus $\mathrm{B}(S)$ is an ideal of $S$. Now let $A$ be an ideal of $S$ and let $a \in A$. If $b \in \mathrm{B}(S)$ then $a$ preserves $b$, so $b=b a z a b$ for some $z \in S$, giving that $b \in A$. Thus $\mathrm{B}(S) \subseteq A$ and it follows that $\mathrm{B}(S)$ is a minimum ideal of $S$. This proves (i).

(ii) $\mathrm{B}(S)$ is a subsemigroup of $S$ by (i). Let $b \in \mathrm{B}(S)$. Since $b$ preserves itself, $H_{b}$ is a subgroup of $S$ by Lemma 3.2 (i). By part (iii) of the same lemma, $H_{b}$ is contained in $\mathrm{B}(S)$. The group inverse of $b$ in $H_{b}$ is therefore an inverse of $b$ in $\mathrm{B}(S)$, so $\mathrm{B}(S)$ is regular.

To complete the proof, we let $a, b \in \mathrm{B}(S)$. Then $(a, b)$ is a mutually preserving pair in $S$, so, by Lemma 3.3, $a b a=a \Leftrightarrow b a b=b$. By [10, Exercise 6, p. 42], $\mathrm{B}(S)$ is 
completely simple.

Part (i) of the above theorem tells us that if, for a semigroup $S, \mathrm{~B}(S)$ is non-empty then $\mathrm{B}(S)$ is the kernel $K(S)$ of $S$ (see $[11$, p. 68]).

If a semigroup $S$ has a universal post-inverse element, then $U$ Post $(S)$ coincides with $\mathrm{B}(S)$, as we now show.

LEMMA 3.8. Let $S$ be a semigroup with a universal post-inverse element. Then UPost $(S)=\mathrm{B}(S)$ and $\mathrm{B}(S)$ is a rectangular band.

Proof: Let $z \in \operatorname{UPost}(S)$. Then $z=z x z$ for all $x \in S$, so $z$ is preserved by every element of $S$, by Lemma 2.2. Thus $z \in \mathrm{B}(S)$, and so UPost $(S) \subseteq \mathrm{B}(S)$.

To prove the reverse inclusion, we again take $z$ to be an element of UPost $(S)$ and let $b \in \mathrm{B}(S)$. Then, since $b$ is preserved by $z$, we have $b=b z t z b$ for some $t \in S$, that is, $b=b z b$. Now let $x$ be any element of $S$. Then $z(b x b) z=z$, and so $b(z b x b z) b=b z b$, that is, $b x b=b$. Thus $b \in \operatorname{UPost}(S)$, giving that $\mathrm{B}(S) \subseteq \operatorname{UPost}(S)$. It follows that $\operatorname{UPost}(S)=\mathrm{B}(S)$.

$\mathrm{B}(S)$ is a completely simple semigroup by Theorem 3.7. It is a band by Lemma 3.4 and so must be a rectangular band.

We note that the subsets UPost $(S)$ and $\mathrm{B}(S)$ of a semigroup are, in general, distinct. For example, a completely simple semigroup $S$ with non-trivial $\mathcal{H}$-classes cannot have a universal post-inverse, by Lemma 3.4, so UPost $(S)=\emptyset$. But $\mathrm{B}(S)=S$ in any completely simple semigroup.

COROLlary 3.9. Let $S$ be a semigroup with a right [left] zero element. Then $\mathrm{B}(S)$ is the set of right [left] zero elements of $S$.

Proof: We have observed earlier that here UPost $(S)$ coincides with the set of right [left] zero elements of $S$. The result follows from Lemma 3.8.

Our final remark in this vein makes use of results given in [6, Exercises 4-6, p. 70]. An element $u$ of a semigroup $S$ is called a zeroid of $S$ if, for each element $a$ of $S$, there exist $x$ and $y$ in $S$ such that $u=a x=y a$. If a semigroup $S$ contains a zeroid then the set $U(S)$ of zeroids of $S$ is a subgroup of $S$ and is the kernel $K(S)$ of $S$ [6, Exercise 6, p. 70].

So let $S$ be a semigroup containing a zeroid. We note first that $U(S) \subseteq \mathrm{B}(S)$. For suppose that $u \in U(S)$. Then $u$ is regular, since it belongs to a subgroup of $S$, and so $u=u t u$ for some $t \in S$. Let $a \in S$. Then $u=(u a) w=z(a u)$ for some $w, z \in S$, so that $u=u t u=(u a w) t(z a u)$. Thus $u$ is preserved by $a$, and it follows that $u \in \mathrm{B}(S)$. This gives that $U(S) \subseteq \mathrm{B}(S)$.

If then, in a semigroup $S, U(S)$ is non-empty, $\mathrm{B}(S)$ is also non-empty and so $U(S)$ $=\mathrm{B}(S)=K(S)$ by a remark above and Theorem 3.7. So, in a semigroup $S$ with a zeroid, $\mathrm{B}(S)$ coincides with $U(S)$ and is a subgroup of $S$. We note that $U(S) \neq \mathrm{B}(S)$ in general, since if $S$ is a rectangular band with more than one element then $U(S)$ is empty but 
$\mathrm{B}(S)=S$.

The author is indebted to the referee for drawing his attention to the subset $U(S)$ in this context.

We now introduce two conditions involving pre- and post-inverses of elements of a semigroup.

Let $S$ be a semigroup and let $a$ be a regular element of $S$. We shall say that

(i) a satisfies the upper inverse condition in $S$ if $\operatorname{Pre}(a) \cap \operatorname{Reg}(S) \subseteq \operatorname{Post}(a)$,

(ii) $a$ satisfies the lower inverse condition in $S$ if $\operatorname{Post}(a) \subseteq \operatorname{Pre}(a)$.

We shall denote the set of regular elements of $S$ that satisfy the upper [lower] inverse condition by $\operatorname{UIC}(S)[\operatorname{LIC}(S)]$.

For an element $a$ of a regular semigroup, the upper and lower inverse conditions become $\operatorname{Pre}(a) \subseteq \operatorname{Post}(a)$ and $\operatorname{Post}(a) \subseteq \operatorname{Pre}(a)$ respectively.

We clearly have that, for $a \in \operatorname{Reg}(S)$,

(i) $a$ satisfies the upper inverse condition

$\Leftrightarrow$ each regular pre-inverse of $a$ is a post-inverse of $a$

$\Leftrightarrow$ each regular pre-inverse of $a$ is an inverse of $a$

$\Leftrightarrow[(\forall b \in \operatorname{Reg}(S)) a=a b a \Rightarrow b=b a b]$,

(ii) $a$ satisfies the lower inverse condition

$\Leftrightarrow$ each post-inverse of $a$ is a pre-inverse of $a$

$\Leftrightarrow$ each post-inverse of $a$ is an inverse of $a$

$\Leftrightarrow[(\forall b \in S) b=b a b \Rightarrow a=a b a]$.

The following result is a straightforward generalisation of $[\mathbf{2}$, Lemma 2.2]. We include a proof for completeness.

Lemma 3.10. Let $S$ be a semigroup and let $a \in \operatorname{Reg}(S)$. Then a satisfies the upper inverse condition if and only if it preserves each of its regular pre-inverses.

PROOF: $a$ satisfies the upper inverse condition

$\Leftrightarrow$ every regular pre-inverse of $a$ is an inverse of $a$

$\Leftrightarrow$ every regular pre-inverse of $a$ is regular in $(S, a)$ (by [8, Corollary 3.3])

$\Leftrightarrow a$ preserves each of its regular pre-inverses.

Lemma 3.10 tells us that in a semigroup $S$ with $\operatorname{Reg}(S) \neq \emptyset$ we have $\operatorname{RRP}(S)$ $\subseteq \mathrm{UIC}(S)$. However, an improved version of this result appears in Theorem 4.2 below.

LEMma 3.11. Let $S$ be a semigroup and let $a \in \operatorname{Reg}(S)$. Then a satisfies the lower inverse condition if and only if it is preserved by each of its post-inverses.

PROOF: Let $a$ satisfy the lower inverse condition and let $b$ be a post-inverse of $a$, that is, let $b=b a b$. Then $a=a b a$, so, by Lemma $2.2, b$ preserves $a$.

Conversely, suppose that $a$ is preserved by each of its post-inverses. Let $b$ be an arbitrary post-inverse of $a$ in $S$. Then $a$ is preserved by $b$, that is, $a$ is regular in $(S, b)$. 
But $a$ is also a pre-inverse of $b$ in $S$. By [8, Corollary 3.3] applied to the (regular) element $b \in S$, the element $a$ is an inverse of $b$ in $S$, so $b$ is an inverse of $a$ in $S$. This shows that $a$ has the lower inverse condition and completes the proof.

COROLlary 3.12. Let $S$ be a semigtoup with $\operatorname{Reg}(S) \neq \emptyset$. Then

$$
\mathrm{B}(S) \subseteq \operatorname{LIC}(S) .
$$

Proof: Let $a \in \mathrm{B}(S)$. Then $a$ is preserved by each element of $S$, so $a \in \operatorname{LIC}(S)$ by Lemma 3.11.

We recall in the next result Lemma 2.3 and Corollary 2.4 of [2].

Lemma 3.13. [2] Let $S$ be a regular semigroup. Then every element of $\operatorname{UIC}(S)$, and hence every element of $\operatorname{RP}(S)$, is maximal in $S$.

Lemma 3.14. Let $S$ be a regular semigroup. Then every element of $\operatorname{LIC}(S)$ is minimal in $S$.

Proof: Let $a \in \operatorname{LIC}(S)$ and let $b \in S$ be such that $b \leqslant a$. Choose $a^{\prime} \in \operatorname{Pre}(a)$. By Lemma 2.1 we have $b=b a^{\prime} b=b a^{\prime} a=a a^{\prime} b$. Now $a^{\prime} b a^{\prime}$ is a post-inverse of $a$, since

$$
\left(a^{\prime} b a^{\prime}\right) a\left(a^{\prime} b a^{\prime}\right)=a^{\prime}\left(b a^{\prime} a\right)\left(a^{\prime} b a^{\prime}\right)=a^{\prime} b\left(a^{\prime} b a^{\prime}\right)=a^{\prime}\left(b a^{\prime} b\right) a^{\prime}=a^{\prime} b a^{\prime} .
$$

But $a$ satisfies the lower inverse condition, so $a^{\prime} b a^{\prime}$ must also be a pre-inverse of $a$. Thus $a\left(a^{\prime} b a^{\prime}\right) a=a$, so $b a^{\prime} a=a$, that is, $b=a$. It follows that $a$ is minimal in $S$, as required.

COROLlary 3.15. In a regular semigroup $S$, every element of $\mathrm{B}(S)$ is minimal in $S$.

Proof: This follows from the preceding lemma and Corollary 3.12 .

\section{TWO REGULARITY-SWITCHING CONDITIONS}

Let $S$ be a semigroup with $\operatorname{Reg}(S) \neq \emptyset$. For $a \in S$, we define the subset $\mathrm{P}(\mathrm{a})$ of $S$ by

$$
\mathrm{P}(a)=\{x \in S: a \text { preserves } x\} .
$$

Similarly, for $a \in \operatorname{Reg}(S)$, we define the subset $\widehat{\mathrm{P}}(\mathrm{a})$ of $S$ by

$$
\widehat{\mathrm{P}}(a)=\{x \in S: x \text { preserves } a\} .
$$

We note at the outset the following:

(i) for $a \in S$, the subsets $\mathrm{P}(a)$ of the semigroup $S$ and $\operatorname{Reg}((S, a))$ of the semigroup $(S, a)$ coincide as subsets of the set $S$;

(ii) for $a \in S$, each element of $\mathrm{P}(a)$ is regular in $S$;

(iii) for $a, b \in S$ with $b$ regular, we have $b \in \mathrm{P}(a) \Leftrightarrow a \in \widehat{\mathrm{P}}(b)$; 
(iv) for $a \in S$, Post $(a) \subseteq \mathrm{P}(a)$, and, for $a \in \operatorname{Reg}(S)$, Pre $(a) \subseteq \widehat{\mathrm{P}}(a)$ (by Lemma $2.2)$;

(v) for $a \in S, a \in \operatorname{RP}(S) \Leftrightarrow \mathrm{P}(a)=\operatorname{Reg}(S)$, and, for $a \in \operatorname{Reg}(S), a \in \mathrm{B}(S)$ $\Leftrightarrow \widehat{\mathrm{P}}(a)=S$. In particular, if $S$ is regular and $a \in S$, then

$$
a \in \mathrm{RP}(S) \Leftrightarrow \mathrm{P}(a)=S, \quad a \in \mathrm{B}(S) \Leftrightarrow \widehat{\mathrm{P}}(a)=S .
$$

For a semilattice $E$ and $e \in E$, we find that

$$
\mathrm{P}(e)=\{f \in E: f \leqslant e\}, \widehat{\mathrm{P}}(e)=\{f \in E: f \geqslant e\} .
$$

If $S$ is a completely simple semigroup and $a \in S$, then $\mathrm{P}(a)=\widehat{\mathrm{P}}(a)=S$.

Now let $S$ be a semigroup and let $a$ be a regular element of $S$. We shall say that

(i) a satisfies the upper switching condition in $S$ if $\widehat{\mathrm{P}}(a) \cap \operatorname{Reg}(S) \subseteq \mathrm{P}(a)$,

(ii) a satisfies the lower switching condition in $S$ if $\mathrm{P}(a) \subseteq \widehat{\mathrm{P}}(a)$.

A regular element of $S$ that satisfies the upper [lower] switching condition will be called an upper [lower] switching element, and we shall denote the set of upper [lower] switching elements by $\mathrm{US}(S)[\mathrm{LS}(S)]$.

For an element $a$ of a regular semigroup $S$, the upper and lower switching conditions become $\widehat{\mathrm{P}}(a) \subseteq \mathrm{P}(a)$ and $\mathrm{P}(a) \subseteq \widehat{\mathrm{P}}(a)$, respectively.

Clearly then, if $S$ is a semigroup and $a \in \operatorname{Reg}(S)$,

(i) $a$ satisfies the upper switching condition $\Leftrightarrow[(\forall b \in \operatorname{Reg}(S)) b$ preserves $a \Rightarrow a$ preserves $b]$,

(ii) $a$ satisfies the lower switching condition $\Leftrightarrow[(\forall b \in S) a$ preserves $b \Rightarrow b$ preserves $a]$.

The following is immediate from the foregoing definitions.

LEMMA 4.1. Let $S$ be a semigroup and let $a \in \operatorname{Reg}(S)$. Then

(i) a satisfies the upper switching condition if and only if it preserves each regular element of $\widehat{\mathrm{P}}(a)$,

(ii) a satisfies the lower switching condition if and only if it is preserved by each element of $\mathrm{P}(a)$.

ThEOREM 4.2 . Let $S$ be a semigroup with $\operatorname{Reg}(S) \neq \emptyset$. Then

$$
\operatorname{RRP}(S) \subseteq \mathrm{US}(S) \subseteq \mathrm{UIC}(S)
$$

If $S$ has a mididentity then $\operatorname{RRP}(S)=\mathrm{US}(S)$.

Proof: Let $a \in \operatorname{RRP}(S)$. Then $a$ is regular and preserves each regular element of $S$. Thus $a \in \mathrm{US}(S)$ by Lemma 4.1 (i), and hence $\operatorname{RRP}(S) \subseteq \mathrm{US}(S)$. 
Next let $a \in \operatorname{US}(S)$. Then $a$ is regular. Let $b$ be a regular pre-inverse of $a$. Then $b$ is a regular element of $\widehat{\mathrm{P}}(a)$, by Lemma 2.2, so $a$ preserves $b$, by Lemma 4.1 (i). Thus we have $a \in \mathrm{UIC}(S)$ by Lemma 3.10, giving that $\mathrm{US}(S) \subseteq \mathrm{UIC}(S)$.

Finally, suppose that $S$ has a mididentity $u$. We may assume that $u$ is idempotent, since the square of a mididentity is an idempotent mididentity [1]. Now let $a \in \operatorname{US}(S)$. Then $a$ is regular and clearly is preserved by $u$. So $a$ preserves $u$, since $a \in \operatorname{US}(S)$. Then Lemma 4.5 of [8] gives that $a \in \operatorname{RRP}(S)$. Thus $\operatorname{US}(S) \subseteq \operatorname{RRP}(S)$, and so $\operatorname{RRP}(S)$ $=\mathrm{US}(S)$.

Theorem 4.2 and Lemma 3.13 now give

COROLLARY 4.3. In a regular semigroup $S$, every element of US $(S)$ is maximal in $S$.

We now consider the 'lower' results corresponding to those in Theorem 4.2.

TheOREM 4.4. Let $S$ be a semigroup with $\operatorname{Reg}(S) \neq \emptyset$. Then $\operatorname{LS}(S)=\operatorname{LIC}(S)$.

PROOF: Let $a \in \operatorname{LS}(S)$. Let $b$ be a post-inverse of $a$. Then $b \in \mathrm{P}(a)$, so $a$ is preserved by $b$, by Lemma 4.1 (ii). Hence $a \in \operatorname{LIC}(S)$ by Lemma 3.11, giving that $\operatorname{LS}(S) \subseteq \operatorname{LIC}(S)$.

To show that $\operatorname{LIC}(S) \subseteq \operatorname{LS}(S)$, we let $a \in \operatorname{LIC}(S)$. Then $a$ is regular and every post-inverse of $a$ is a pre-inverse of $a$. To show that $a \in \operatorname{LS}(S)$, we must show that the condition

$(\forall b \in S) \quad a$ preserves $b \Rightarrow b$ preserves $a$

holds. So let $b \in S$ be such that $a$ preserves $b$. Then $b=b a z a b$ for some $z \in S$. This gives that $z a b=z a b . a . z a b$, that is, $z a b$ is a post-inverse of $a$. So $z a b$ is a pre-inverse of $a$, that is, $a=a(z a b) a$.

Similarly, $b=b a z a b$ gives $b a z=b a z . a . b a z$, so $a=a(b a z) a$. But $a=a w a$ for some $w \in S$, since $a$ is regular, and so $a=(a b a z a) w(a z a b a)$, giving that $b$ preserves $a$. Thus $a \in \operatorname{LS}(S)$ and so $\operatorname{LIC}(S) \subseteq \operatorname{LS}(S)$. This completes the proof.

Combining this result with Corollary 3.12, we get

COROLlary 4.5 . Let $S$ be a semigroup with $\operatorname{Reg}(S) \neq \emptyset$. Then

$$
\mathrm{B}(S) \subseteq \operatorname{LS}(S)=\operatorname{LIC}(S)
$$

We recall [5] that an $E$-inversive semigroup may be characterised as a semigroup $S$ such that Post(s) is non-empty for all $s \in S$. The class of $E$-inversive semigroups includes the class of regular semigroups and has been widely studied (see Mitsch's survey [15]).

We know that a semigroup has a regular element if and only if it has an idempotent. So, if $a$ is an element of a semigroup $S$, then $\operatorname{Reg}((S, a))$ is non-empty if and only if $E((S, a))$ is non-empty, that is, $\mathrm{P}(a)$ is non-empty if and only if Post $(a)$ is non-empty (using [8, Lemma 3.1 (iii)]). We have thus proved the following result.

THEOREM 4.6. Let $S$ be a semigroup with $\operatorname{Reg}(S) \neq \emptyset$. Then $S$ is $E$-inversive if and only if $\mathrm{P}(s)$ is non-empty for each $s \in S$. 
We immediately have

COROLlary 4.7. If a semigroup $S$ is such that $\mathrm{B}(S)$ is non-empty, then $S$ is E-inversive.

The converse of the last result is false, as may be seen by taking $S$ to be the bicyclic semigroup. Here $S$ is regular and hence $E$-inversive, but $\mathrm{B}(S)$ is empty. (See Example 6.2 below).

We next show that the inclusion $\mathrm{B}(S) \subseteq \mathrm{LS}(S)$ of Corollary 4.5 becomes an equality if $S$ is $E$-inversive.

THEOREM 4.8. Let $S$ be an E-inversive semigroup. Then

$$
\mathrm{B}(S)=\mathrm{LS}(S)=\operatorname{LIC}(S) .
$$

Proof: Clearly $\operatorname{Reg}(S) \neq \emptyset$. By Corollary 4.5 it is enough to show that $\operatorname{LIC}(S)$ $\subseteq \mathrm{B}(S)$. So let $a \in \operatorname{LIC}(S)$. Let $x$ be an arbitrary element of $S$. We shall show that $x$ preserves $a$. Let $y \in \operatorname{Post}(x a)$. Then $y=y(x a) y$. This gives $y x=(y x) a(y x)$, so $y x$ is a post-inverse of $a$. Therefore $y x$ is a pre-inverse of $a$, since $a \in \operatorname{LIC}(S)$, so that $a=a(y x) a$.

Similarly, let $z \in \operatorname{Post}(a x)$. Then $z=z(a x) z$. This gives $x z=(x z) a(x z)$, so that $x z$ is a post-inverse of $a$. Thus $x z$ is a pre-inverse of $a$, that is, $a=a(x z) a$.

But $a=a c a$ for some $c \in S$, since $a$ is regular, so $a=(a x z a) c(a y x a)$. Thus $x$ preserves $a$. It follows that $a \in \mathrm{B}(S)$, so that $\mathrm{LIC}(S) \subseteq \mathrm{B}(S)$, as required.

Corollary 4.9. Let $S$ be a regular semigroup. Then

$$
\mathrm{B}(S)=\mathrm{LS}(S)=\operatorname{LIC}(S) .
$$

\section{MAXIMAL AND MINIMAL ELEMENTS IN A REGULAR SEMIGROUP}

In this section we restrict ourselves to regular semigroups. Here we have $\operatorname{RP}(S)$ $\subseteq \mathrm{US}(S) \subseteq \mathrm{UIC}(S)$ by Theorem 4.2, and the elements of these subsets are all maximal in $S$ by Lemma 3.13. We shall consider another kind of maximal element in $S$, and examine the relationship between the set of those elements and the subsets above.

Similarly, we know that $\mathrm{B}(S)=\operatorname{LS}(S)=\operatorname{LIC}(S)$, by Corollary 4.9, and Lemma 3.14 gives that all the elements of $\mathrm{B}(S)$ are minimal in $S$.

So let $S$ be a regular semigroup and let $\operatorname{Min}(S)[\operatorname{Max}(S)]$ denote the set of minimal [maximal] elements in $S$. We begin by showing that $\mathrm{B}(S)$ accounts for all of $\operatorname{Min}(S)$. Of course, if $S$ has a zero element 0 , then $\operatorname{Min}(S)=\{0\}$ and $\mathrm{B}(S)=\{0\}$ (as noted in section 3).

THEOREM 5.1. Let $S$ be a regular semigroup. Then $\operatorname{Min}(S)=\mathrm{B}(S)$.

Proof: By Lemma 3.14 it is enough to show that $\operatorname{Min}(S) \subseteq \operatorname{LIC}(S)$. So let $a \in \operatorname{Min}(S)$. Let $b \in \operatorname{Post}(a)$, that is, let $b a b=b$. Then $a b a=(a b) a=a(b a)$, where 
$a b$ and $b a$ are idempotents, giving that $a b a \leqslant a$. Thus $a b a=a$, since $a$ is minimal, that is, $b \in \operatorname{Pre}(a)$. It follows that $a \in \operatorname{LIC}(S)$, and we have that $\operatorname{Min}(S) \subseteq \operatorname{LIC}(S)$, as required.

Before discussing maximal elements we need to recall the following definition [8, Section 2]. If $H$ is a subset of a semigroup $S$, we say that $a \in S$ is weakly cancellable on $H$ if the condition

$$
(\forall x, y \in H) \quad x a=y a \text { and } a x=a \dot{y} \Rightarrow x=y
$$

holds. We shall denote the set of elements of $S$ that are weakly cancellable on $H$ by $\mathrm{WC}(H)$. (Elements of $\mathrm{WC}(S)$ are usually called weak cancellation elements in the literature.)

Lemma 5.2. Let $S$ be a semigroup with $\operatorname{Reg}(S) \neq \emptyset$ and let $a \in S$. Then $a$ is weakly cancellable on $\mathrm{P}(a)$.

Proof: Let $x, y \in \mathrm{P}(a)$. Then $x$ and $y$ are regular and $a$ preserves each of them. By Lemma 3.1, we have $x a \mathcal{R} x \mathcal{L} a x, y a \mathcal{R} y \mathcal{L} a y$. Suppose now that $x a=y a$ and $a x=a y$. This gives $x \mathcal{H} y$. By Green's Lemma [11, Lemma 2.2.1], $\rho_{a} \mid L_{x}$ is a bijection from $L_{x}$ to $L_{x a}$. But $x \rho_{a}=y \rho_{a}$, so we must have $x=y$.

THEOREM 5.3. Let $S$ be a regular semigroup. Then $\mathrm{RP}(S) \subseteq \mathrm{WC}(S) \subseteq \operatorname{UIC}(S)$.

Proof: Let $a \in \operatorname{RP}(S)$. Then $\mathrm{P}(a)=S$, so, by Lemma 5.2, $a$ is weakly cancellable on $S$, that is, $a \in \mathrm{WC}(S)$. Thus $\mathrm{RP}(S) \subseteq \mathrm{WC}(S)$.

Next let $a \in \mathrm{WC}(S)$. The element $a$ is, of course, regular in $S$. So, in order to show that $a \in \operatorname{UIC}(S)$, we must verify that the condition

$$
(\forall b \in S) a=a b a \Rightarrow b=b a b
$$

holds. So let $b \in S$ be such that $a=a b a$. Then $a(b a b)=a(b),(b a b) a=(b) a$, giving that $b a b=b$, since $a$ is weakly cancellable on $S$. Thus $a \in \operatorname{UIC}(S)$, and so $\operatorname{WC}(S) \subseteq \operatorname{UIC}(S)$, as required.

Lemma 3.13 now gives

COROLLARY 5.4. In a regular semigroup $S$, every element of $\mathrm{WC}(S)$ is maximal in $S$.

For a regular semigroup $S$, let $\mathfrak{M}(S)=\{\operatorname{RP}(S), \operatorname{US}(S), \mathrm{WC}(S), \mathrm{UIC}(S), \operatorname{Max}(S)\}$. So the members of $\mathfrak{M}(S)$ are the subsets of maximal elements discussed above. The 
inclusions that we have established between these are given by the following diagram.

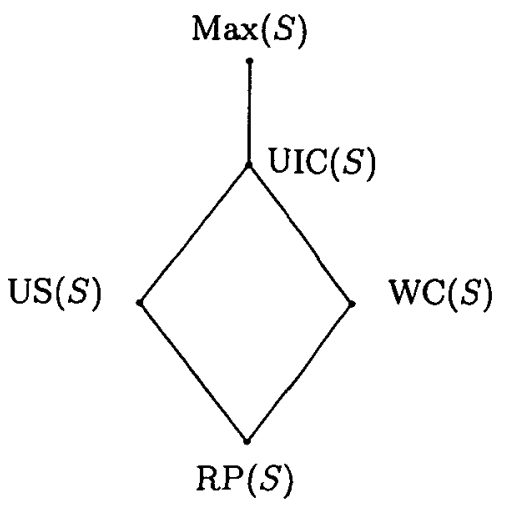

The examples given in the next section will show that, in general, no other inclusions are possible.

We conclude this section with some results on completely simple semigroups.

THEOREM 5.5. Let $S$ be a semigroup such that $\operatorname{Reg}(S) \neq \emptyset$. Then

$$
S \text { is completely simple } \Leftrightarrow \mathrm{B}(S)=S \text {. }
$$

Proof: If $S$ is completely simple then, as remarked in Section 3, we have $\mathrm{B}(S)=S$, If $\cdot \mathrm{B}(S)=S$ then $S$ is completely simple by Theorem 3.7 .

The results given in [10, Exercise 6, p. 42] are well-known and tell us that, for a regular semigroup $S$, the following are equivalent:
(i) $S$ is completely simple;
(ii) $\mathrm{WC}(S)=S$;
(iii) $\operatorname{UIC}(S)=S$.

Theorem 5.6 below gives some characterisations of a completely simple semigroup, and the two noted above appear amongst them. We have, however, already used the implication (iii) $\Rightarrow$ (i) above in the build-up to this theorem (for [8, Theorem 4.4] and for Theorem 3.7 above), so the equivalence of (i) and (iii) above is not being proved here. Nonetheless, for the purposes of Theorem 5.6 we shall retain $\mathrm{UIC}(S)$ as a member of the set $\mathfrak{M}(S)$ defined earlier.

THEOREM 5.6. The following are equivalent for a regular semigroup $S$ :

(i) $S$ is completely simple;

(ii) each member of $\mathfrak{M}(S)$ coincides with $S$;

(iii) some member of $\mathfrak{M}(S)$ coincides with $S$.

Proof: Let $S$ be a regular semigroup. We prove that (i) $\Rightarrow$ (ii) $\Rightarrow$ (iii) $\Rightarrow$ (i). Suppose that $S$ is completely simple. Then $\operatorname{RP}(S)=S$, so, by the inclusions indicated 
in the diagram above, each member of $\mathfrak{M}(S)$ coincides with $S$. Thus (i) $\Rightarrow$ (ii). The implication (ii) $\Rightarrow$ (iii) is obviously true. Suppose, finally, that (iii) holds. Then $\operatorname{Max}(S)$. $=S$, so the partial order on $S$ is trivial. It follows that $\operatorname{Min}(S)=S$, so that $\mathrm{B}(S)=S$, by Theorem 5.1 . Thus, by Theorem $3.7, S$ is completely simple.

\section{EXAMPLES}

In this final section of the paper we consider some useful examples. We shall use $\subset$ to denote strict containment between subsets.

EXAMPLE 6.1. $[3,12]$ Let $S$ be the Rees matrix semigroup $\mathcal{M}^{0}(\{1\} ;\{1,2\},\{1,2\} ; P)$, where

$$
P=\left(\begin{array}{ll}
1 & 1 \\
1 & 0
\end{array}\right) .
$$

Here $S$ is a regular semigroup with distinct elements

$$
e=(1,1,1), f=(1,1,2), g=(2,1,1), h=(2,1,2), 0,
$$

where 0 is the zero element of $S$. There is just one non-idempotent element in $S$, namely $h$. We quickly find that the four non-zero elements are maximal in $S$, so that $\operatorname{Max}(S)$ $=\{e, f, g, h\}, \operatorname{Min}(S)=\{0\}$. Clearly, we have that $\mathrm{B}(S)=\{0\}$.

It is shown in [12] that $\operatorname{RP}(S)=\{e\}$, and we easily find that $\mathrm{WC}(S)=\{e\}$ also.

Using Lemma 3.1, we see that

$$
\mathrm{P}(e)=S, \mathrm{P}(f)=\{e, f, 0\}, \mathrm{P}(g)=\{e, g, 0\}, \mathrm{P}(h)=\{e, 0\}, \mathrm{P}(0)=\{0\} .
$$

It follows from these that

$$
\widehat{\mathrm{P}}(e)=\{e, f, g, h\}, \widehat{\mathrm{P}}(f)=\{e, f\}, \widehat{\mathrm{P}}(g)=\{e, g\}, \widehat{\mathrm{P}}(h)=\{e\}, \widehat{\mathrm{P}}(0)=S .
$$

Thus $\widehat{\mathrm{P}}(a) \subseteq \mathrm{P}(a)$ for all non-zero elements $a \in S$, so that $e, f, g, h \in \mathrm{US}(S)$. Since

$$
\operatorname{Max}(S)=\{e, f, g, h\} \subseteq \mathrm{US}(S) \subseteq \operatorname{UIC}(S) \subseteq \operatorname{Max}(S),
$$

we have that $\operatorname{US}(S)=\operatorname{UIC}(S)=\operatorname{Max}(S)=\{e, f, g, h\}$.

We also find that $e$ is a universal pre-inverse for $S$, so $\operatorname{UPre}(S)=\operatorname{RP}(S)=\{e\}$, by Lemma 3.6. Thus, to sum up, we have

$$
\operatorname{UPre}(S)=\operatorname{RP}(S)=\mathrm{WC}(S) \subset \operatorname{US}(S)=\operatorname{UIC}(S)=\operatorname{Max}(S) .
$$

We may conclude from this that, in general, for a regular semigroup $S, \operatorname{RP}(S)$ is distinct from $\operatorname{US}(S)$ and $\mathrm{WC}(S)$ is distinct from $\operatorname{UIC}(S)$.

Finally, this example shows (as does Example 6.3 below) that a non-band semigroup may have a universal pre-inverse element. 
EXAMPLE 6.2. The bicyclic semigroup $S$ is constructed as follows: let $N$ be the set of non-negative integers and take $S=N \times N$, with multiplication

$$
(m, n)(p, q)=(m-n+t, q-p+t)
$$

where $t=\max (n, p) \quad(m, n, p, q \in N)$. (For an account of this semigroup see [6, 11, 13]). It is an inverse monoid with group of units $G=\{(0,0)\}$, right unit subsemigroup $P=\{(0, n): n \in N\}$, and left unit subsemigroup $Q=\{(m, 0): m \in N\}$.

Since $S$ has an identity element, we have $\operatorname{RP}(S)=G[8$, p. 380] and, the identity element being a mididentity, we have $\mathrm{RP}(S)=\mathrm{US}(S)$, by Theorem 4.2. So $\mathrm{RP}(S)$ $=\mathrm{US}(S)=G$.

The partial order on $S$ is calculated in [13, Section 3.4], and we have that, for $(m, n),(p, q) \in S$

$$
(m, n) \leqslant(p, q) \Leftrightarrow m=p+a \text { and } n=q+a \text { for some } a \in N .
$$

We easily see now that $\operatorname{Max}(S)=P \cup Q$. Also, the elements of $P[Q]$ are right [left] cancellable on $S$, and so are weakly cancellable on $S$. Thus

$$
\operatorname{Max}(S)=P \cup Q \subseteq \mathrm{WC}(S) \subseteq \mathrm{UIC}(S) \subseteq \operatorname{Max}(S)
$$

and it follows that $\mathrm{WC}(S)=\operatorname{UIC}(S)=\operatorname{Max}(S)=P \cup Q$. So we have

$$
\mathrm{RP}(S)=\mathrm{US}(S) \subset \mathrm{WC}(S)=\mathrm{UIC}(S)=\operatorname{Max}(S)
$$

We thus see that, in general, for a regular semigroup $S, \operatorname{RP}(S)$ is distinct from $\mathrm{WC}(S)$, and $\operatorname{US}(S)$ is distinct from $\operatorname{UIC}(S)$. Also, we note that $\mathrm{US}(S) \subset \mathrm{WC}(S)$ for the semigroup of the present example, and $\mathrm{WC}(S) \subset \mathrm{US}(S)$ for the semigroup of Example 6.1 , and we may conclude that, in general, for a regular semigroup $S$, neither one of the subsets WC $(S), \operatorname{US}(S)$ is contained in the other.

We may note that the bicyclic semigroup $S$, not being a band, has no universal pre-inverse element, by Lemma 3.6. Also, $S$ has no minimal element, so $\mathrm{B}(S)$ is empty. Finally, as pointed out by the referee, this is an example where $\mathrm{B}(S)$ is empty but the kernel $K(S)$ of $S$ is non-empty (since $K(S)=S$ here).

The following interesting example was devised by McAlister [14] and proved to be useful in [9] and [12]. We recall that, if $T$ is a regular semigroup and, $\mathcal{M}(T ; I, \Lambda ; P)$ is a Rees matrix semigroup over $T$ then the set of regular elements in $\mathcal{M}(T ; I, \Lambda ; P)$ forms a regular semigroup [14]. This latter semigroup is denoted by $\mathcal{R} \mathcal{M}(T ; I, \Lambda ; P)$.

EXAMPLE 6.3. [14] Let $T$ be the chain semilattice $\{1, a, b, 0\}$ with $1>a>b>0$. Let $I=\Lambda=\{1,2\}$ and let $P$ be the $2 \times 2$ matrix

$$
P=\left(\begin{array}{ll}
1 & a \\
b & 0
\end{array}\right)
$$


Let $S=\mathcal{R M}(T ; I, \Lambda ; P)$. Then $S$ is a regular semigroup with eleven elements, namely

$$
\begin{array}{llll}
(1,1,1) & & & \\
(1, a, 1), & (2, a, 1), & & \\
(1, b, 1), & (2, b, 1), & (1, b, 2), & (2, b, 2), \\
(1,0,1), & (2,0,1), & (1,0,2), & (2,0,2) .
\end{array}
$$

The element $(2, b, 2)$ is the only non-idempotent in $S$. We easily see that $S$, under the usual partial order, consists of four disjoint chains:

$$
\begin{aligned}
(1,1,1)>(1, a, 1)>(1, b, 1) & >(1,0,1), \\
(2, a, 1)>(2, b, 1) & >(2,0,1), \\
(1, b, 2) & >(1,0,2), \\
(2, b, 2) & >(2,0,2) .
\end{aligned}
$$

Thus we have

$$
\begin{aligned}
\operatorname{Max}(S) & =\{(1,1,1),(2, a, 1),(1, b, 2),(2, b, 2)\} \\
\operatorname{Min}(S) & =\{(1,0,1),(2,0,1),(1,0,2),(2,0,2)\} .
\end{aligned}
$$

We find that the element $(1,1,1)$ is a pre-inverse of every element of $S$ and that it has just one pre-inverse, namely itself. We conclude from this that UPre $(S)=\{(1,1,1)\}$ and also that $\operatorname{Post}((1,1,1))=S$ and $\operatorname{Pre}((1,1,1))=\{(1,1,1)\}$. Since Pre $((1,1,1))$ $\subseteq \operatorname{Post}((1,1,1))$, we have that $(1,1,1) \in \mathrm{UIC}(S)$. Further, if $x \in S, x \neq(1,1,1)$, then $(1,1,1)$ is a pre-inverse but not a post-inverse of $x$, so $x \notin \operatorname{UIC}(S)$. Thus $\operatorname{UIC}(S)$ $=\{(1,1,1)\}$. We now have that $\operatorname{UPre}(S)=\operatorname{UIC}(S)=\{(1,1,1)\}$ and, since $\operatorname{UPre}(S)$ $\subseteq \mathrm{RP}(S)$, it follows that

$$
\mathrm{UPre}(S)=\mathrm{RP}(S)=\mathrm{US}(S)=\mathrm{WC}(S)=\operatorname{UIC}(S)=\{(1,1,1)\} .
$$

In this example, therefore, we have that $\operatorname{UIC}(S) \subset \operatorname{Max}(S)$.

Finally, we have, by Theorem 5.1, that $\mathrm{B}(S)=\operatorname{Min}(S)$. Also, the element $(1,0,1)$ is a universal post-inverse for $S$, so, by Lemma 3.8, we have UPost $(S)=\mathrm{B}(S)$. Thus

$$
\operatorname{UPost}(S)=\mathrm{B}(S)=\operatorname{Min}(S) \text {. }
$$

Our final example shows that, in general, for a semigroup $S$ we have $\mathrm{B}(S) \neq \operatorname{LIC}(S)$. EXAMPLE 6.4. Let $A=\langle a\rangle$ be an infinite monogenic semigroup and let $S=A^{1}$. Then $S$ has just one regular element, namely 1 . Also, $\operatorname{Pre}(1)=\operatorname{Post}(1)=\{1\}$, giving that $1 \in \operatorname{LIC}(S)$, so that $\operatorname{LIC}(S)=\{1\}$. Clearly $\mathrm{B}(S)=\emptyset$, and so we have $\mathrm{B}(S) \subset \operatorname{LIC}(S)$. 


\section{REFERENCES}

[1] J.E. Ault, 'Semigroups with midunits', Trans. Amer. Math. Soc. 190 (1974), 375-384.

[2] T.S. Blyth and J.B. Hickey, 'RP-dominated regular semigroups', Proc. Roy. Soc. Edinburgh 99A (1984), 185-191.

[3] T.S. Blyth and R. McFadden, 'On the construction of a class of regular semigroups', J.Algebra 81 (1983), 1-22.

[4] S.J. Boyd, M. Gould and A. Nelson, 'Interassociativity of semigroups', in Proceedings of the Tennessee Topology Conference (Nashville, TN, 1996) (World Sci. Publishing, River Edge, N.J., 1997), pp. 33-51.

[5] F. Catino and M.M. Miccoli, 'On semidirect product of semigroups', Note Mat. 9 (1989), 189-194.

[6] A.H. Clifford and G.B. Preston, The algebraic theory of semigroups, Vol. 1 (Amer. Math. Soc., Providence, R.I., 1961).

[7] R.E. Hartwig, 'How to partially order regular elements', Math. Japon. 25 (1980), 1-13.

[8] J.B. Hickey, 'Semigroups under a sandwich operation', Proc. Edinburgh Math. Soc. 26 (1983), 371-382.

[9] J.B. Hickey, 'On variants of a semigroup', Bull. Austral. Math. Soc. 34 (1986), 447-459.

[10] P.M. Higgins, Techniques of semigroup theory (Oxford University Press, Oxford, 1992).

[11] J.M. Howie, Fundamentals of semigroup theory (Clarendon Press, Oxford, 1995)).

[12] T.A. Khan and M.V. Lawson, 'Variants of regular semigroups', Semigroup Forum 62 (2001), 358-374.

[13] M.V. Lawson, Inverse semigroups. The theory of partial symmetries (World Sci. Publishing, River Edge, N.J., 1998).

[14] D.B. McAlister, 'Regular Rees matrix semigroups and regular Dubreil-Jacotin semigroups', J. Austral. Math. Soc. Ser. A 31 (1981), 325-336.

[15] H. Mitsch, 'Introduction to $E$-inversive semigroups', in Semigroups (Braga, 1999) (World Sci. Publishing, River Edge, N.J., 2000), pp. 114-135.

[16] K.S.S. Nambooripad, 'The natural partial order on a regular semigroup', Proc. Edinburgh Math. Soc. 23 (1980), 249-260.

[17] M. Yamada, 'A note on middle unitary semigroups', Kodai Math. Sem. Rep. 7 (1955), 49-52.

Department of Mathematics

University of Glasgow

Glasgow G12 8QW

Scotland

e-mail: jbh@maths.gla.ac.uk 